\title{
Selecting and Making the Most of Online Webinars in Plastic Surgery: A New Initiative
}

\author{
Abizer Kapadia' Mahesh Nair ${ }^{2}$ Vikramaditya Salvi ${ }^{3}$
}

\author{
${ }^{1}$ Department of Plastic Surgery, NMC Royal Hospital, Dubai, UAE \\ ${ }^{2}$ Department of Plastic Surgery, Sunrise Hospital, Kerala, India \\ ${ }^{3}$ Department of Plastic Surgery, Phi Aesthetics Center, India
}

Indian J Plast Surg:2020;53:293-297

Our lives have certainly changed in a way that we would have never imagined. There is no more running away from the fact that this pandemic or lockdown does not affect us. It is real and it is here to stay. To make the best use of this lockdown time, educating and enhancing our knowledge while sitting at home seems to be the most appropriate option in these tough times.

There has been a sudden boost in the number of available online platforms for hosting and managing online meetings and conferences, which ushers in a new era, one in which we do not have to meet physically: welcome to the world of webinars. In simple words, any seminar or meeting conducted over the Internet is termed as a webinar. Choosing the right one could be a task for some of us, who possess limited technical knowledge, especially if we are the ones organizing the event rather than simply attending them.

We, a group of young plastic surgeons, started a platform named "Young Plastic Surgeons (YPS)-India, Webinar Series. The sole motive of this nonsponsored, nonprofit venture was to connect, educate, learn, and discuss with the younger generation of plastic surgeons in India. YPS-India is a virtual group on social media platforms, which is connected via smaller closed groups, with the intent of helping our colleagues via case discussion and distribution of educational opportunities. Although the group is named YPS, it is available to all belonging to our fraternity. We have organized biweekly meets, wherein we invited speakers from India and beyond. These speakers consisted of established practicing consultant plastic surgeons to those new in practice and starting out. This is still an ongoing process.

The most popular topics discussed were as follows: some offbeat and some mainstream topics to make things interesting-Liposuction and its techniques, essential softwares (mobile and PC) for the quintessential plastic surgeon, facial anatomy-Botox and fillers, and establishing an esthetic practice or a solo microsurgery service, to name a few.

Having used various platforms, we have compiled a list of the most noteworthy ones. As per standard norms, Internet speed of more than 2 Mbps speed is required for any platform to run smoothly (livestreaming ones such as YouTube live may require even more, a minimum of 4 Mbps.
Address for correspondence Dr. Abizer Kapadia, MS, MCh, Department of Plastic Surgery, NMC Royal Hospital, Dubai, UAE (e-mail: Abizer.kapadia@gmail.com).

The online platforms we came across were as follows:

1. Zoom-It is an American communications technology company. Certainly, the most popular platform available in the market today!. Has options of both, requiring password and enabling "waiting room" feature, giving more control to the host. It has an inbuilt recording feature which can be automated for all meetings ( - Fig. 1).

2. GoToMeeting-Web-hosted, cloud-based subscription service for online meeting and video conferencing. An American venture again. A good, easy, and simple platform having most of the features that match Zoom's in every way ( - Fig. 2 ).

Offshoot-GoToWebinar (highly professional platform with cloud storage and added security-suitable for large companies with confidential content sharing).

3. Webex-Cisco Webex is an American Company that develops and sells app-based web meeting and video conferencing applications. Offers similar platform to zoom and others, with reliability of Cisco systems, and also offers screen sharing and no time limit.

4. Google Meet-It is a video conferencing app and is Google's answer to Zoom. It is the business-oriented version of Google's Hangouts platform and is suitable for businesses of all sizes. It integrates seamlessly with $G$ Suite versions of Google Calendar and Gmail and helps you connect using various preinstalled Google apps.

5. BeThereGlobal-This is a UK-based company which aids in conducting online events, webcasting, conference, and event recording. No free versions to this. But is one of the safest platforms available to conduct large scale online meetings/webinars.

6. Livestorm-A French company. This is a slightly different software, as it is a browser-based online web conferencing software used to share real-time live streams. Can be used to power remote live meetings, product demos, sales webinars, online lessons, onboarding sessions, and more. It integrates as an extension in your browser.

7. Youtube Live: It is a livestreaming platform best designed for one-on-one discussions or debates with limited number of participants, which sends a message across in a
DOI https://doi.org/

10.1055/s-0040-1715539

ISSN 0970-0358.
License terms

(1) (1) $\Theta \circledast$ 


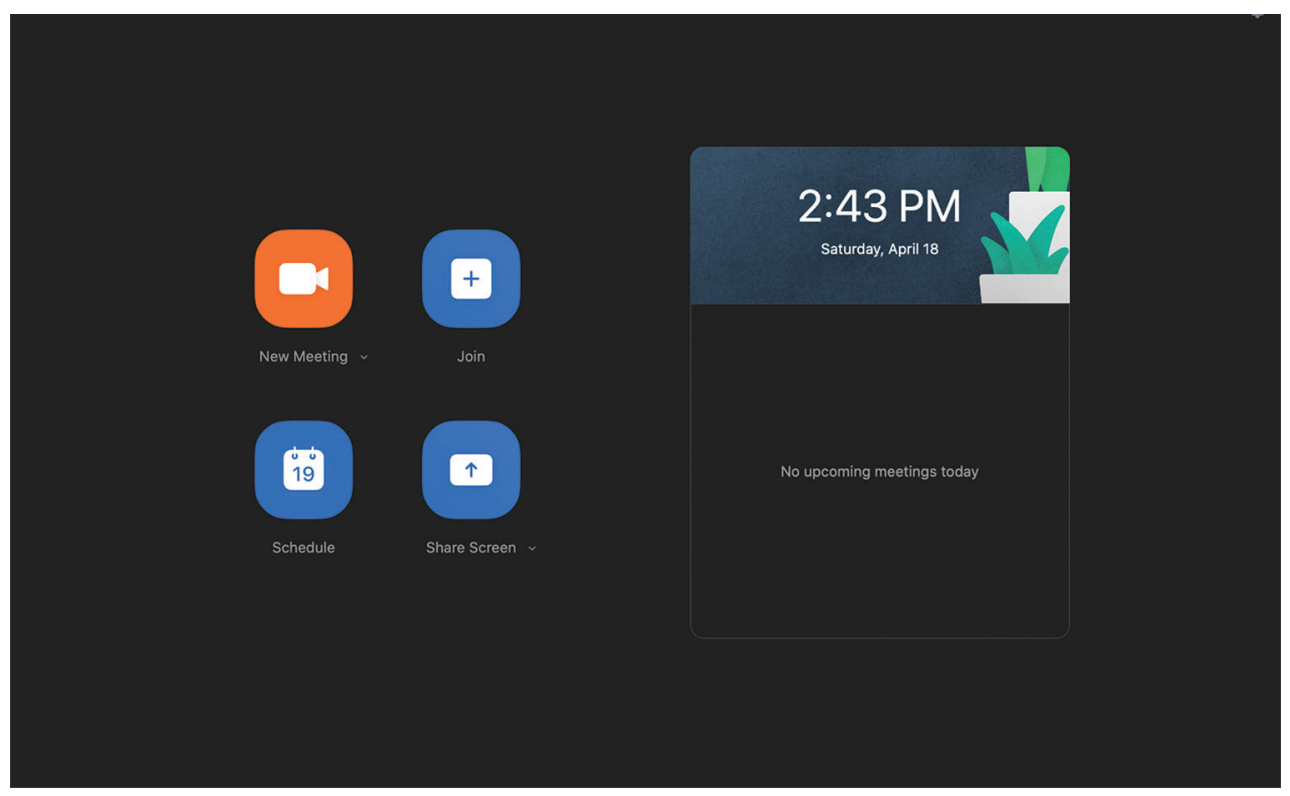

Fig. 1 Zoom application main window.

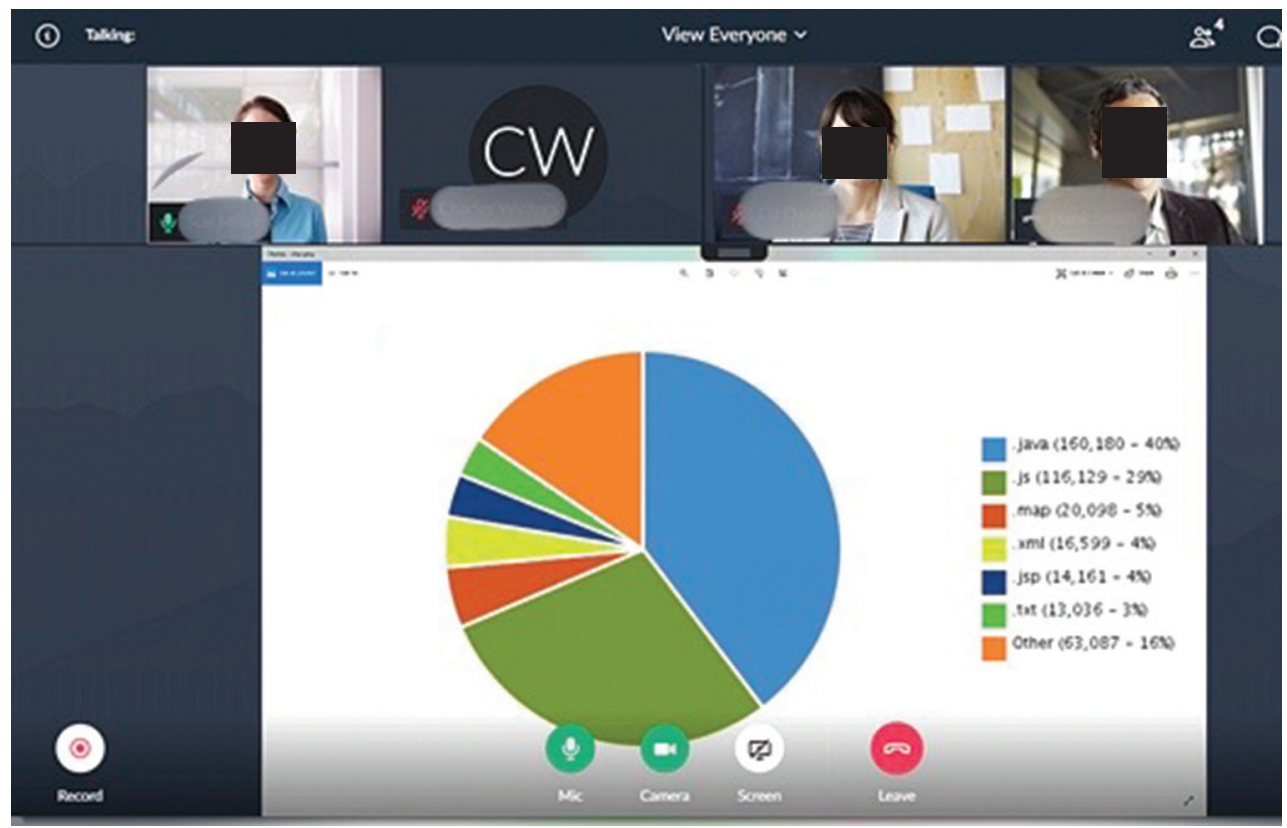

Fig. 2 GoToMeeting-meeting interface.

nonformal/casual way. In our opinion, it is not a viable option to conduct educational webinar with formal teaching. Although free, it is plagued by ads. Lot of content qualification restrictions are required.

8. Microsoft Teams-A unified communication and collaboration platform which combines workplace chat, video meetings, file storage, and application integration. The service integrates with the Office 365 subscription. It is a more formal, complex, and a business/company-oriented platform, which is somewhat complicated to understand (-Table 1).
There is a plethora of other paid and unpaid platforms available, which have been in use for various purposes. We have particularly omitted Facebook meet/live and Instagram live, since these are more informal platforms, rather outright social media platforms, and may not convey the same seriousness merited by the content of an academic webinar/meeting, especially one incorporating continued medical education (CME) credit points. Attendees cannot be restricted/verified even if it is a closed group.Moreover, there is no option of screen sharing, and live session is restricted to 1 hour at a time. We did come across some other platform 


\begin{tabular}{|c|c|c|c|c|c|c|c|c|c|c|}
\hline 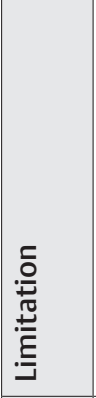 & 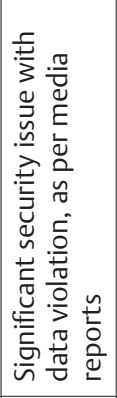 & 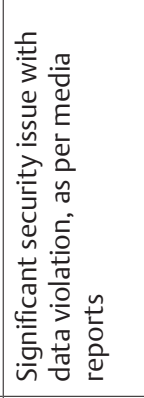 & 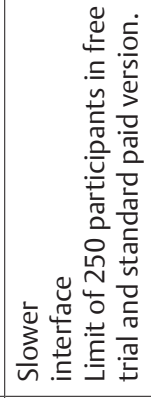 & 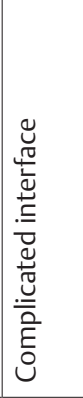 & 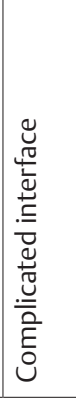 & 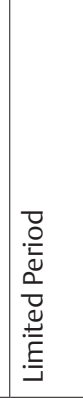 & 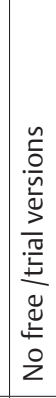 & 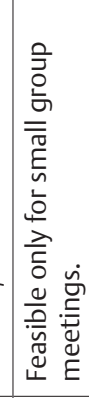 & 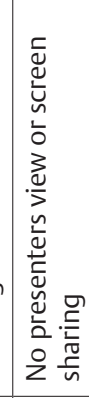 & 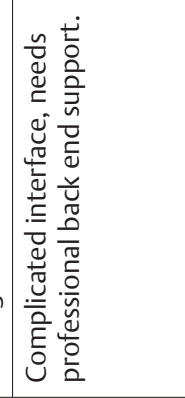 \\
\hline 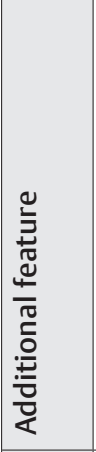 & 1 & 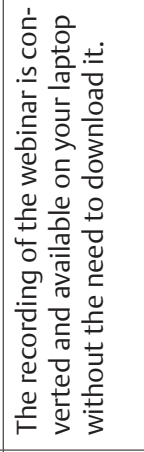 & 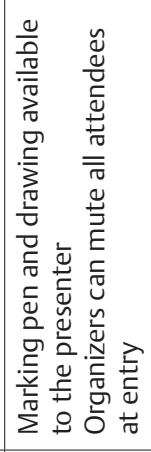 & 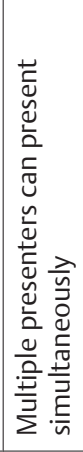 & 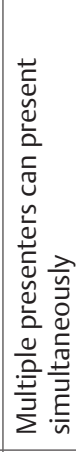 & 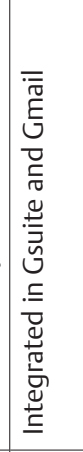 & 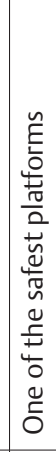 & 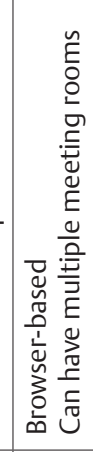 & 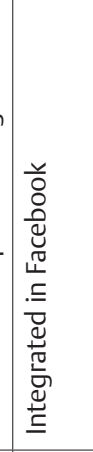 & 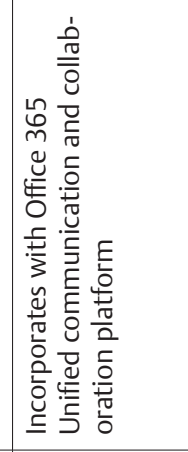 \\
\hline 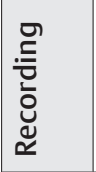 & $\stackrel{\check{y}}{\succ}$ & $\stackrel{\check{\nu}}{\nu}$ & $\stackrel{\check{\nu}}{\nu}$ & $\stackrel{\check{\nu}}{\nu}$ & $\stackrel{\check{\nu}}{\nu}$ & z & $\stackrel{\check{\nu}}{\nu}$ & $\stackrel{\tilde{\nu}}{\nu}$ & 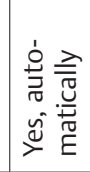 & $\stackrel{\check{\nu}}{\nu}$ \\
\hline 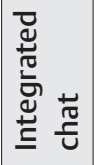 & $\stackrel{\check{y}}{\succ}$ & $\stackrel{\tilde{\nu}}{\rightleftharpoons}$ & $\stackrel{\check{\nu}}{\check{\nu}}$ & 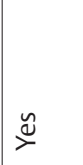 & $\stackrel{\check{\nu}}{\check{0}}$ & $\stackrel{\check{\nu}}{\nu}$ & $\stackrel{\check{\nu}}{\rightleftharpoons}$ & $\stackrel{\check{\nu}}{\nu}$ & $\stackrel{\check{\nu}}{\nu}$ & $\stackrel{\check{\nu}}{\rightleftharpoons}$ \\
\hline 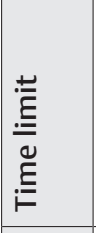 & 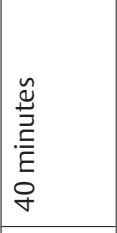 & 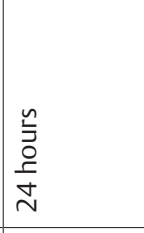 & 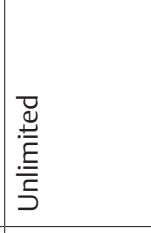 & 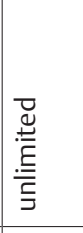 & 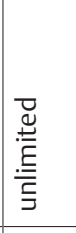 & 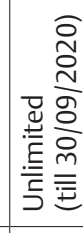 & 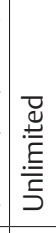 & 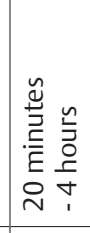 & 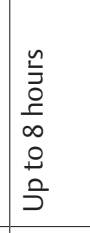 & 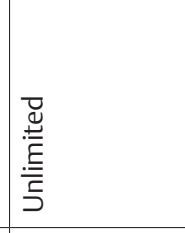 \\
\hline 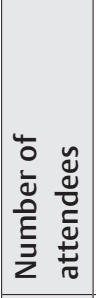 & 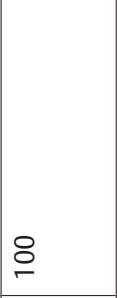 & 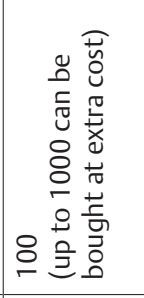 & 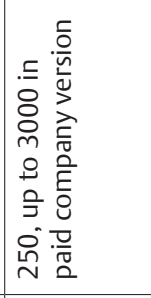 & 8 & $\begin{array}{l}8 \\
0 \\
0 \\
m \\
\dot{1} \\
\stackrel{0}{1}\end{array}$ & 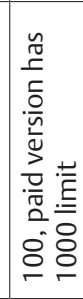 & 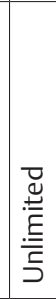 & $\begin{array}{l}8 \\
\frac{0}{1} \\
\end{array}$ & 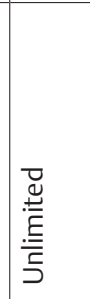 & 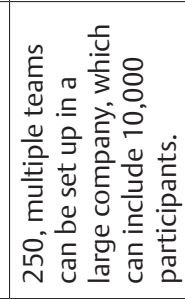 \\
\hline 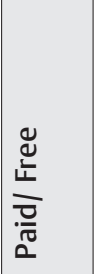 & $\begin{array}{l}\text { むँ } \\
\text { 岑 }\end{array}$ & 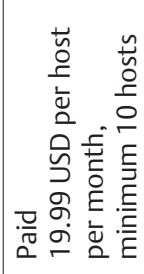 & 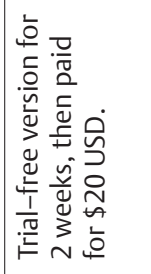 & 㫕 & 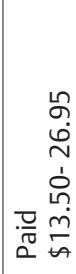 & 荧 & $\begin{array}{l}\frac{\pi}{\pi} \\
\check{0}\end{array}$ & 总 & 总 & $\frac{\pi}{\bar{\pi}}$ \\
\hline 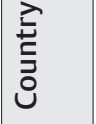 & 芯 & 芯 & 芯 & 芯 & $\stackrel{\sim}{د}$ & 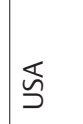 & כ & 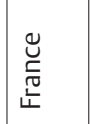 & 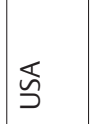 & 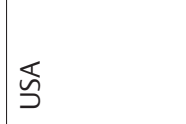 \\
\hline 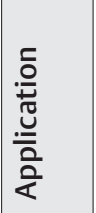 & E & 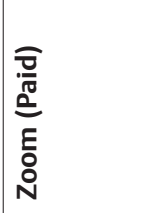 & 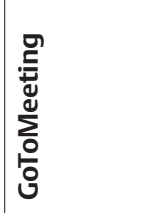 & खेّ & 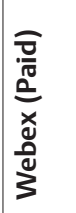 & 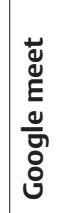 & 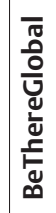 & E & 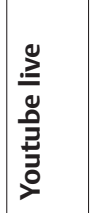 & 总 \\
\hline
\end{tabular}


from India, namely, saynamaste.in and JioMeet $\beta$ version. These platforms, again, were not tried by us personally and have mainly been used for nonacademic/informal family meetings. Detailed elaboration of these and many other available platforms is beyond the scope of this paper.

An important issue of security and protection of confidentiality arises when medical education, especially plastic surgery, is discussed and more so when it is recorded. Security of data and copyright issues are addressed by issuing a disclaimer/undertaking before a webinar is conducted. These are again subjective to the organizer. An example of such disclaimer/warning/undertaking is what we incorporated in YPS registration forms, asking the participants to agree to nonplagiarism norms, no distribution of material in any form, along with ethical use of data, and protection of confidentiality of patients ( - Fig. 3). We also try to ensure that the

\section{Young Plastic Surgeons (YPS) Webinar Contact Information}

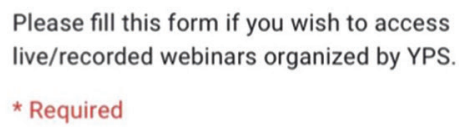

Name *

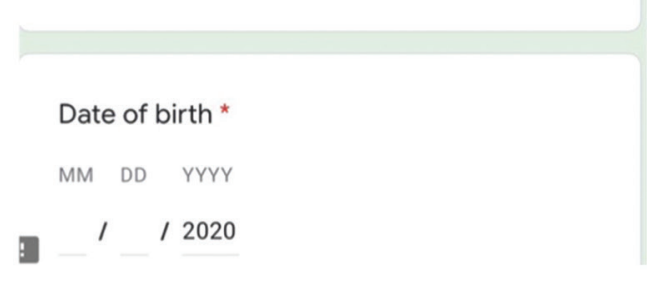

Address *

Your answer

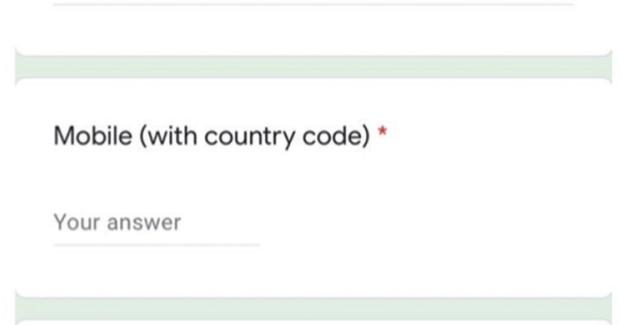

City of practice *

Your answer

Qualification(s) *
$\square$ M.Ch.
$\square$ DNB

APSI Membership (if yes, mention membership number in 'other' column) *

$\square$ Yes

No

Other:

IAAPS Membership ( if yes, mention membership number in 'other' column) *

\section{Yes}

No

Other:

ISAPS Membership ( if yes, mention membership number in 'other' column) *

$\square$ Yes

No

Other:

Other International Society Membership (if yes, mention membership number in 'other' column)*
Yes
No
Other:

Fig. 3 Disclaimer and consent used by YPS Webinar Series-the attendee/ participant has to fill a registration form with personal details and agree to the terms and conditions before attending the webinar. 
link for the webinars is circulated in closed groups (of plastic surgeons alone). Recording in any form and its distribution invites copyright issues, plagiarism issues, etc. Hence, a disclaimer/undertaking has to be procured before airing sensitive content (as considered by the presenter). This safeguards the organizer/presenter legally. It is up to the discretion of the organizers and presenters, whether and which, content should be recorded and made available later or not. It is a subjective matter and has nothing to do with the platforms used as such. Elucidating all the terms and conditions is beyond the scope of this paper. Terms and conditions of each webinar keep changing, depending on the organizer and presenter preferences, and they should be read and understood by the viewers prior to logging in, as recommended.Since the webinar culture is in its infancy, most of the rules and regulations, rather etiquettes, are just being formulated.

Some of the common ones/unanimously accepted world over, which are incorporated in YPS webinars are as follows:

a. Mute microphone at arrival.

b. Preferably turn off webcam.

c. Use raise hand feature to draw attention.

d. Use full log in name/valid email address and other credentials to $\log$ in (most platforms take this information before they allow a $\log$ in).

e. Type questions/comments only in the chat box provided. Use good language. Avoid unconventional acronyms/short forms/social media language.

f. Dress appropriately as per social norms.

Lately, e-Education and webinar culture has also helped CME in plastic surgery training. PG clinics can be successfully conducted on such platforms and live 1:1 interaction can be achieved. Numerous institutes in India are already conducting daily e-journal clubs, case presentations, virtual grand rounds, APLAST series, etc. using these platforms.

What you should look for in a webinar platform:

1. Affordable.

2. Safe.

3. Should allow sufficient number of participants.

4. Should have an adequate time allowance as per requirements.

5. Should be relatively easy to use for professionals and nonprofessionals alike.

6. Should preferably integrate into the existing browser, avoiding the need to download more applications/ softwares
7. Should have recording function.

8. Should be able to schedule a meeting and have option of recurring meetings.

9. Should integrate with the scheduler/calendar of your smart device.

10. Desirable to have pen/brush function (e.g., GoToMeeting) to edit/highlight presentation content on-the-air.

11. Desirable to have cloud space for storage of recorded data with ease of retrieval.

12. Adequate caution needs to be addressed to incorporate antiplagiarism and protect patient's confidentiality by keeping the recording safe and circulated only among a desired closed section of people (this feature is not possible with platforms such as FB live/Insta live).

To summarize, we firmly believe, the culture of online webinars/platforms has ushered in a new age of learning and education with a new era, which will not be limited to the lockdown period or COVID-19 pandemic. Since the technological advances are user friendly enough for a layman, in the long term, it will serve as a cost-effective modality of discussion, knowledge sharing, meeting, conferencing, e-learning, all with a touch of thorough professionalism

There are indications from some of the major plastic surgery societies planning to conduct a complete virtual conference, spanning over a couple of days with the entire setup, including didactic lectures, live operative streams and participants interaction being conducted in the near future. This would be available from the comfort of your home, providing essential CME credits and at a much lower overall cost. In the future, these platforms in one form or another could make way into many other sectors such as banking, real estate registrations, DIY classes, etc. The possibilities are limitless.

Our picks would be GoToMeeting, Zoom and GoogleMeet, since these are widely being used and offer significant ease of operability. Number of participants, time limits, budget constraints and content protection would be the most important features for consideration while selecting the most suitable online platforms.

Quoting Sir William Osler, "The best preparation for tomorrow is to do today's work superbly well."

\section{Conflicts of Interest}

None declared. 